\title{
ON A GENERALIZATION OF THE CORONA PROBLEM
}

\section{GRAZIANO GENTILI and DANIELE C. STRUPPA}

\author{
Scuola Normale Superiore \\ Piazza dei Cavalieri, 7 \\ 56100 Pisa, Italy \\ (Received May 7, 1985)
}

ABSTRACT. Let $g, f_{1}, \ldots, f_{m} \in H^{\infty}(\Delta)$. We provide conditions on $f_{1}, \ldots, f_{m}$ in order that $|g(z)| \leqslant\left|f_{1}(z)\right|+\ldots+\left|f_{m}(z)\right|$, for all $z$ in $\Delta$, imply that $g$, or $g^{2}$, belong to the ideal generated by $f_{1}, \ldots, f_{m}$ in $H^{\infty}$.

KEY WORDS AND PHRASES. Corona problem, congenial functions.

1980 AMS SUBJECT CLASSIFICATION CODE. 30D55, $30 D 50$.

\section{INTRODUCTION.}

Let $H(\Delta)=\mathrm{H}$ be the space of all holomorphic functions on $\Delta=\{z \in \mathbb{C}:|z|<1\}$, and let $\mathrm{H}^{\infty}(\Delta)=\mathrm{H}^{\infty}$ be the subspace of all bounded functions of $\mathrm{H}(\Delta)$. Let $\mathrm{f}_{1}, \ldots, \mathrm{f}_{\mathrm{m}}$ be functions in $\mathrm{H}^{\infty}$ and let $\mathrm{g \in \textrm {H } ^ { \infty }}$ satisfy the following condition:

$$
|g(z)| \leqslant\left|f_{1}(z)\right|+\ldots+\left|f_{m}(z)\right| \quad(\text { any } z \in \Delta) \text {. }
$$

As a generalization of the corona problem (which was first solved by Carleson [1]) it is natural to ask if (1.1) implies that $g$ belongs to the ideal $I_{H} \infty\left(f_{1}, \ldots, f_{m}\right)$ generated in $\mathrm{H}^{\infty}$ by $\mathrm{f}_{1}, \ldots, \mathrm{f}_{\mathrm{m}^{\prime}}$ i.e. if (1.1) implies the existence of $\mathrm{g}_{1}, \ldots, \mathrm{g}_{\mathrm{m}}$ in $\mathrm{H}^{\infty}$ such that, on $\Delta$,

$$
g=f_{1} g_{1}+\ldots+f_{m} g_{m}
$$

Rao, [2], has shown that the answer to this question is negative in general. On the other hand Wolff (see [3], th. 2.3) has proved that (1.1) implies that $g^{3}$ belongs to $I_{H^{\infty}}\left(f_{1}, \ldots, f_{m}\right)$. The question whether $(1.1)$ implies the existence of $g_{1}, \ldots g_{m}$ in $H^{\infty}$ such that

$$
\mathrm{g}^{2}=\mathrm{f}_{1} \mathrm{~g}_{1}+\ldots+\mathrm{f}_{\mathrm{m}} \mathrm{g}_{\mathrm{m}}
$$

is still open, as Garnett has pointed out ([4], problem 8.20).

In this work we obtain some results on this generalized corona problem, making use of techniques which appear in the theory of $A_{p}$ spaces, the spaces of entire functions with growth conditions introduced by Hörmander [5].

With the same aim of Berenstein and Taylor [6] in $A_{p}$ ' we introduce in $H^{\infty}$ the notion of jointly invertible functions (definition 3 ) and prove that if $f_{1}, \ldots, f_{m}$ are jointly invertible, condition (1.1) implies that $g$ belongs to $I_{H^{\infty}}\left(f_{1} \ldots \ldots f_{m}\right.$ ) (proposition 5$)$. We also prove that if the ideal $I_{H^{\infty}}\left(f_{1}, \ldots, f_{m}\right)$ contains a weakly invertible 
function having simple interpolating zeroes (see [3]), then again (1.1) Implies that g belongs to $I_{H^{\circ}}\left(f_{1}, \ldots, f_{m}\right)$ (theorem 6$)$.

Finally, in the same spirit of Kelleher and Taylor [7] we introduce the notion of congeniality for m-tuples of functions in $\mathrm{H}^{\infty}$, and give a partial answer to the problem posed by Garnett $([4])$ : we prove that if $\left(f_{1}, \ldots, f_{m}\right) \in\left(H^{\infty}\right)^{m}$ is congenıal, then (1.1) implies $g^{2} \in I_{H^{\infty}}\left(f_{1}, \ldots, f_{m}\right)$ (theorem 8 ).

2. WEAK INVERTIBILITY.

We first study some conditions under which (1.1) implies that $g \in I_{H^{\infty}}\left(f_{1}, \ldots, f_{m}\right)$. DEFINITION 1. A function $f$ in $H^{\infty}(\Delta)$ is called weakly invertible if there exists a Blaschke product $B$ such that $f(z)=B(z) \tilde{f}(z)$ ( $z$ in $\Delta$ ) with $\tilde{f}$ invertible in $H^{\infty}$.

The reason for this definition is the following simple criterion of divisibility for functions in $\mathrm{H}^{\infty}$.

PROPOSITION 2. Let $f \in \mathrm{H}^{\infty}$. Then $\mathrm{f}$ is weakly invertible if, and only if, for all $\mathrm{g} \in \mathrm{H}^{\infty}$ the fact that $\mathrm{g} / \mathrm{f} \in \mathrm{H}$ implies $\mathrm{g} / \mathrm{f} \in \mathrm{H}^{\infty}$.

PROOF. Suppose $f$ is weakly invertible: then there exists a Blaschke product $B$ such that $f(z)=B(z) \tilde{f}(z)$, with $\tilde{f}$ invertible in $H^{\infty}$. Since $g / f$ is holomorphic and since $B$ contains exactly the zeroes of $f$, it follows that $g / B \in H$; however, since $B$ is $a$ Blaschke product, $g / B \in H$ implies, $[8]$, that $g / B \in H^{\infty}$. Since $1 / \tilde{f} \in H^{\infty}$ one has $g / f=(g / B)(1 / \tilde{f})$, i.e. $g / f \in H^{\infty}$. Conversely, suppose that for all $g \in H^{\infty}$ such that $g / f \in H$, it follows $g / f \in H^{\infty}$. Write $f(z)=B(z) \tilde{f}(z)$, where $B$ is the Blaschke product of all the zeroes of $f$ (see [8]). Then $B / f$ is holomorphic on $\Delta$ and therefore $1 / \tilde{f}$ must belong to $H^{\infty}$.

An extension of the notion of weak invertibility to m-tuples of functions in $\mathrm{H}^{\infty}$ is given by the following definition, analogous to the one given by Berenstein and Taylor for the spaces $A_{p}$ in [6].

DEFINITION 3. The functions $f_{1}, \ldots, f_{m} \in H^{\infty}$ are called jointly invertible if the ideal generated by $f_{1}, \ldots, f_{m}$ in $H^{\infty}$ coincides with $I_{10 c}\left(f_{1}, \ldots, f_{m}\right)=\left\{g \in H^{\infty}(\Delta)\right.$ : for any $z \in \Delta$, there exists a neighborhood $U$ of $z$ and $\lambda_{1}, \ldots, \lambda_{m}$ in $H(U)$ such that $g=\lambda_{1} f_{1}+\ldots+\lambda_{m} f_{m}$ on $U$ \}.

In view of Cartan's theorem $B$, it follows immediately that $f_{1}, \ldots, f_{m}$ are jointly invertible if, and only if, $I_{H^{\infty}}\left(f_{1}, \ldots, f_{m}\right)=I_{H}\left(f_{1}, \ldots, f_{m}\right)$, the latter being the ideal generated by $f_{1}, \ldots, f_{m}$ in $H(\Delta)$. As a consequence of the corona theorem, all m-tuples $f_{1}, \ldots, f_{m}$ in $H^{\infty}$ for which there exists $\delta>0$ such that $\left|f_{1}(z)\right|+\ldots+\left|f_{m}(z)\right| \geqslant \delta$ for all z in $\Delta$, are jointly invertible $\left(I_{H}=I_{H}^{\infty}{ }^{\infty} H^{\infty}\right)$. More generally one has:

PROPOSITION 4. Let $b \in H^{\infty}$ be weakly invertible, and let $f_{1}(z)=b(z) \tilde{f}_{1}(z), \ldots, f_{m}(z)=$ $=b(z) \tilde{f}_{m}(z)$, for $\tilde{f}_{1}, \ldots, \tilde{f}_{m}$ in $H^{\infty}$ such that $\left|\tilde{f}_{1}(z)\right|+\ldots+\left|\tilde{f}_{m}(z)\right| \geqslant \delta>0$ for some $\delta$ and all $z$ in $\Delta$. Then $f_{1}, \ldots, f_{m}$ are jointly invertible.

PROOF. Let $g \in H^{\infty}$ belong to $I_{H}\left(f_{1}, \ldots, f_{m}\right)$. There exist $\lambda_{1}, \ldots, \lambda_{m}$ in $H(\Delta)$ such that

$$
g(z)=\lambda_{1}(z) f_{1}(z)+\ldots \lambda_{m}(z) f_{m}(z) \quad(\text { all } z \in \Delta)
$$

i.e., for all $z$ in $\Delta$,

$$
g(z)=b(z)\left[\lambda_{1}(z) \tilde{f}_{1}(z)+\ldots+\lambda_{m}(z) \tilde{f}_{m}(z)\right] .
$$

Since $b$ is invertible, and $g / b \in H$, it follows that $\tilde{g}=g / b=\lambda_{1} \tilde{f}_{1}+\ldots+\lambda_{m} \tilde{f}_{m} \in H^{\infty}$. By the corona theorem, then, it follows that there are $h_{1} \ldots, h_{m}$ in $H^{\infty}$ such that

$$
\tilde{g}(z)=h_{1}(z) \tilde{f}_{1}(z)+\ldots+h_{m}(z) \tilde{f}_{m}(z),
$$

therefore

$$
g(z)=\tilde{g}(z) b(z)=h_{1}(z) f_{1}(z)+\ldots+h_{m}(z) f_{m}(z)
$$


and the assertion is proved.

Let now $f_{1}, \ldots, f_{m}, g \in H^{\infty}(\Delta)$, and suppose that (1.1) holds. It is well known, [2], that in general $(1.1)$ does not imply that $g \in I_{H^{\infty}}\left(f_{1}, \ldots, f_{m}\right)$. However, (1.1) certainly implies that $g \in I_{10 c}\left(f_{1}, \ldots, f_{m}\right)$ and hence

PROPOSITION 5. Let $\mathrm{f}_{1}, \ldots, \mathrm{f}_{\mathrm{m}}$ be jointly invertible. Then if $\mathrm{g}$ satisfies condition (1.1), it follows that $g \in I_{H^{\infty}}\left(f_{1}, \ldots, f_{m}\right)$.

A different situation in which (1.1) implies that $g \in I_{H^{\infty}}\left(f_{1}, \ldots, f_{m}\right)$ occurs when at least one of the $f_{j}$ 's, say $f_{1}$, is weakly invertible and has simple zeroes which form an interpolating sequence ([3]); this happens, for example, when $f_{1}$ is an interpolating Blaschke product with simple zeroes ([3]). Indeed, following an analogous result proved in [7] for the space of entire functions of exponential type, one has:

THEOREM 6. Let $\mathrm{f}_{1}, \ldots, \mathrm{f}_{\mathrm{m}} \in \mathrm{H}^{\infty}$, and suppose $\mathrm{f}_{1}$ is weakly invertible with simple, interpolating zeroes. Then if ${\mathrm{g} \in \mathrm{H}^{\infty}}^{\infty}$ satisfies condition (1.1) it follows that $\mathrm{g}$ belongs to $I_{H^{\infty}}\left(f_{1}, \ldots, f_{m}\right)$.

PROOF. Choose $a_{i j} \in \mathbb{C}, i=2, \ldots m, j \geqslant 1$, such that for $\left\{z_{j}\right\}=\left\{z \in \Delta: f_{1}(z)=0\right\}$ it is $\left|a_{i j}\right|=1$ and $a_{i j} f_{i}\left(z_{j}\right) \geqslant 0$. Define now $b_{i j} \in \mathbb{C}(i, j$ as before $)$ by

$$
b_{i j}=\left\{\begin{array}{l}
0 \text { if } f_{2}\left(z_{j}\right)=\ldots=f_{m}\left(z_{j}\right)=0 \\
a_{i j} g\left(z_{j}\right) /\left(\left|f_{2}\left(z_{j}\right)\right|+. .+\left|f_{m}\left(z_{j}\right)\right|\right) \text { otherwise. }
\end{array}\right.
$$

By (1.1) it follows $\left|b_{i j}\right| \leqslant 1(a l l i, j)$, and since $\left\{z_{j}\right\}$ is interpolating, one finds $h_{2}$, $\ldots, h_{m}$ in $H^{\infty}$ such that $h_{i}\left(z_{j}\right)=b_{i j}$. Therefore the function $h=g-\left(h_{2} f_{2}+\ldots+h_{m} f_{m}\right)$ belongs to $H^{\infty}$ and vanishes at each $z_{j}$. The simplicity of the zeroes of $f_{1}$ shows that $f / f_{1} \in H$, and the invertibility of $f_{1}$ implies $h / f_{1}=h_{1} \in H^{\infty}$. The thesis now follows, since $g=f_{1} h_{1}+$ $+\ldots+f_{m} h_{m}$.

It is worthwhile noticing that the hypotheses of proposition 5 and Theorem 6 are not comparable. Consider, indeed, the following conditions on $f_{1}, \ldots, f_{m} \in H^{\infty}$ :

$\left(C_{1}\right) f_{1}, \ldots, f_{m}$ are jointly invertible.

$\left(c_{2}\right)$ there exists $j(1 \leqslant j \leqslant m)$ such that $f_{j}$ is invertible, with an interpolating sequence of zeroes, all of which are simple.

Then $\left(c_{1}\right)$ does not imply $\left(c_{2}\right)$ : take $m=1$ and $f_{1}$ weakly invertible with non-simple zeroes. on the other hand, also $\left(C_{2}\right)$ does not imply $\left(C_{1}\right)$ : consider $f_{1}$ invertible with simple interpolating zeroes $\left\{z_{n}\right\}$; let $\mathrm{f}_{2} \in \mathrm{H}^{\infty}$ be a function such that $\mathrm{f}_{2}\left(z_{n}\right)=1 / n$ (such a function certainly exists since $\left\{z_{n}\right\}$ is an interpolating sequence); now $f_{1}$ and $f_{2}$ have no common zeroes, and hence $1 \in I_{\text {loc }}\left(f_{1}, f_{2}\right)$; however $1 \notin I_{H^{\infty}}\left(f_{1}, f_{2}\right)$ since if $1=\lambda_{1} f_{1}+\lambda_{2} f_{2}$, then it is $\lambda_{2}\left(z_{n}\right)=n$, i.e. $\lambda_{2} \notin H^{\infty}$. Therefore the pair $\left(f_{1}, f_{2}\right)$ satisfies $\left(C_{2}\right)$ but not $\left(C_{1}\right)$.

3. CONGENIALITY.

In this section we describe a class of m-tuples of functions in $H^{\infty}(\Delta)$, for which condition (1.1) implies that $g^{2} \in I_{H^{\infty}}\left(f_{1}, \ldots, f_{m}\right)$.

DEFINITION 7. An m-tuple $\left(f_{1}, \ldots, f_{m}\right)$ of functions in $H^{\infty}$ is called congenial if, for all $i, j=1, \ldots, m$,

$$
\left(f_{i} f_{j}^{\prime}-f_{j} f_{i}^{\prime}\right) /\|f\|^{2}\left\|f^{\prime}\right\| \text { belongs to } L^{\infty}(\Delta),
$$

where $|f(z)|^{2}=\left|f_{1}(z)\right|^{2}+\ldots+\left|f_{m}(z)\right|^{2}, \|\left. f^{\prime}(z)\right|^{2}=\left|f_{i}^{\prime}(z)\right|^{2}+\ldots+\left|f_{m}^{\prime}(z)\right|^{2}$, and $f_{i}^{\prime}=\partial f_{i} / \partial z$. 
Notice that the class of congenial m-tuples is not empty. Indeed, one might consider pairs $f_{1}, f_{2}$ in $H^{\infty}$ which, at their common zeroes, satisfy some simple conditions on their vanishing order easily deducible from Definition 7. For example, one can ask that $f_{1}\left(z_{0}\right)=f_{2}\left(z_{0}\right)=0, f_{2}^{\prime}\left(z_{0}\right) \neq 0, f_{1}^{\prime}\left(z_{0}\right)=0$. As a partial answer to problem 8.20 in [4], we prove the following

THEOREM 8. Let $f_{1}, \ldots, f_{m}, g \in H^{\infty}(\Delta)$, and suppose $\left(f_{1}, \ldots, f_{m}\right)$ be congenial. If $g$ satisfies (1.1), then $g^{2} \in I_{H^{\infty}}\left(f_{1}, \ldots, f_{m}\right)$, i.e. there are $g_{1}, \ldots, g_{m}$ in $H^{\infty}$ such that (on $\Delta$ )

$$
g^{2}(z)=f_{1}(z) g_{1}(z)+\ldots+f_{m}(z) g_{m}(z)
$$

PROOF. We mainly follow the proof due to Wolff, [3], of the fact that (1.1) implies that $g^{3} \in I_{H^{\infty}}$. We can assume $\left\|f_{j}\right\|_{\infty} \leqslant 1, \mid g \|_{\infty} \leqslant 1$, and $f_{j}, g \in H(\bar{\Delta}) \quad(j=1, \ldots, m)$. Put $\psi_{j}=g \bar{f}_{j} /|f|^{2}$ $\left(\psi_{j}\right.$ is bounded and $c^{\infty}$ on $\left.\bar{\Delta}\right)$ and consider the differential equation

$$
\partial b_{j, k} / \partial \bar{z}=\psi_{j} \partial \psi_{k} / \partial \bar{z}=g^{2} G_{j, k} \quad(1 \leqslant j, k \leqslant m)
$$

for

$$
G_{j, k}=\bar{f}_{j} \sum_{\ell} f_{\ell}\left(\overline{\left.f_{\ell^{f}}^{f_{k}^{\prime}-f_{k} f_{\ell}^{\prime}}\right)} /|f|^{6} .\right.
$$

If solutions $b_{j, k} \in L^{\infty}$ exist, then clearly $g_{j}=g \psi_{j}+\sum_{k}\left(b_{j, k}-b_{k, j}\right) f_{k} \in H^{\infty}$ and (3.1) holds (indeed $\bar{\partial} g_{j}=0$ and $g_{j}$ is bounded on $\Delta$ ). In order to prove that $(3.2$ ) admits a solution in $L^{\prime \infty}$ it is enough to show that $\left|g^{2} G_{j, k}\right|^{2} \log (1 /|z|) d x d y$ and $\partial\left(g^{2} G_{j, k}\right) / z$ are carleson measures for $1 \leqslant j, k \leqslant m$.

As far as $\left|g^{2} G_{j, k}\right|^{2} \log (1 /|z|) d x d y$ is concerned, notice that, by the congeniality of $\left(f_{1}, \ldots, f_{m}\right)$, it is

$$
\left|g^{2} G_{j, k}\right|^{2} \leqslant|g|^{4}\left|\bar{f}_{j}\right|^{2} \mid \sum_{\ell} f_{\ell}\left(\left.\overline{\left.f_{\ell_{k}^{\prime}}^{f_{k}^{\prime}-f_{k} f_{\ell}^{\prime}}\right)}\right|^{2} /|f|^{12} \leqslant c\left|f^{\prime}\right|^{2} .\right.
$$

On the other hand,

$$
\partial\left(g^{2} G_{j, k}\right) / \partial z=2 g g^{\prime} G_{j, k}+g^{2} \partial G_{j, k} / \partial z_{i}
$$

again by the congeniality of $\left(f_{1}, \ldots, f_{m}\right)$, one has

$$
\begin{aligned}
& \left|g g^{\prime} G_{j, k}\right| \leqslant|g|\left|g^{\prime}\right|\left|\bar{f}_{j}\right| \mid \sum_{\ell} f_{\ell}\left(\left.\overline{f_{\ell^{\prime} j^{\prime}-f_{k^{\prime}} f_{\ell^{\prime}}}}|/| f\right|^{6} \leqslant c\left(\left|g^{\prime}\right|^{2}+\left\|f^{\prime}\right\|^{2}\right) / f \mid \leqslant\right. \\
& \leqslant c\left(\left|g^{\prime}\right|^{2} /|g|+\left|f^{\prime}\right|^{2} / \| f \mid\right),
\end{aligned}
$$

and

$$
\begin{aligned}
& \left|g^{2} \partial G_{j, k} / \partial z\right|=|g|^{2} \cdot\left|f_{j}\right|\left|\sum_{\ell} \bar{f}_{\ell} f_{\ell}^{\prime}\right| \cdot\left|\sum_{\ell} f_{\ell} \overline{\left(f_{\ell} f_{k}^{\prime}-f_{k} f_{\ell}^{\prime}\right.}\right| /|f|^{8}+ \\
& +|g|^{2}\left|\bar{f}_{j}\right| /|f|^{2} \cdot\left(\left|\sum_{\ell} f_{\ell}^{\prime}\left(\frac{\ell}{\left(f_{\ell} f_{k}^{\prime}-f_{k} f_{\ell}^{\prime}\right.}\right)\right| /\left.\left|f \|^{4}+2\right| \sum_{\ell} f_{\ell^{\prime}} \bar{f}_{\ell}|| \sum_{\ell} f_{\ell}\left(\overline{f_{\ell} f_{k}^{\prime}-f_{k} f_{\ell}^{\prime}}\right)|/| f\right|^{6}\right) \leqslant \\
& \leq c \sum_{\ell}\left|f_{\ell}\right|^{2} /\left|f_{\ell}\right| \text {. }
\end{aligned}
$$

This concludes the proof.

ACKNOWLEDGIENT. Theauthors wish to thank Professor Carlos A. Berenstein for reading a preliminary version of this paper. They also gladlyacknowledge the Ministero P.I. of the Italian Government and the University of Maryland for their financial support. 


\section{REFERENCES}

1. CARLESON, L. Interpolation by Bounded Analytic Functions and the Corona Problem, Ann. of Math. (2) 76 (1963), 547-559.

2. RAO, K.V.R. On a Generalized Corona Problem, J. Anal. Math. 18(1967), 277-278.

3. GARNetT, J.B. Bounded AnalyticFunctions, Academic Press, New York, 1981.

4. BARTH, K.F., BRANNAN, D.A. AND HAYMAN, W.K. Research Problems in Complex Analysis, Bul1. London Math. Soc. 16 (1984), 490-517.

5. HORMANDER, L. Generators for Some Rings of Analytic Functions, Bull. Amer. Math. Soc. 73 (1967), 943-949.

6. BERENSTEIN, C.A. and TAYLOR, B.A. Interpolation Problems in $\mathbb{C}^{\mathrm{n}}$ with Applications to Harmonic Analysis, J. Anal. Math. 38 (1980), 188-254.

7. KELLEHER, J.J. and TAYLOR, B.A. FInitely Generated Ideals in Rings of Analytic Functions, Math. Ann. 193 (1971), 225-237.

8. HOFFMAN, K. Banach Spaces of Analytic Functions, Prentice Hall Inc., Englewood Cliffs, 1962. 


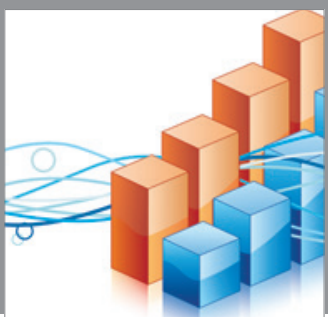

Advances in

Operations Research

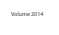

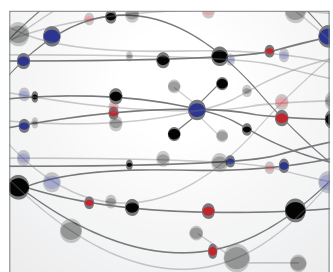

\section{The Scientific} World Journal
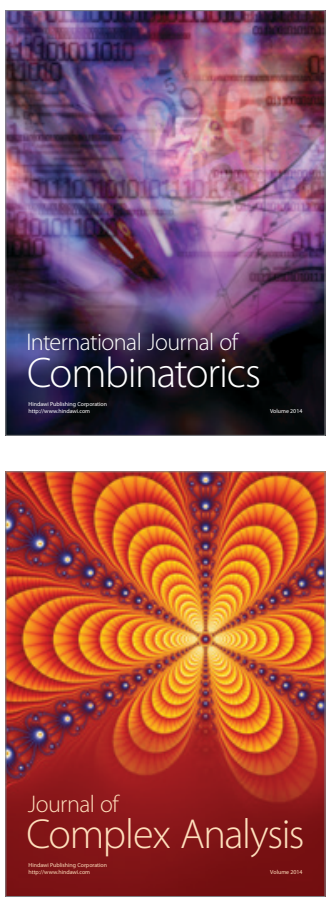

International Journal of

Mathematics and

Mathematical

Sciences
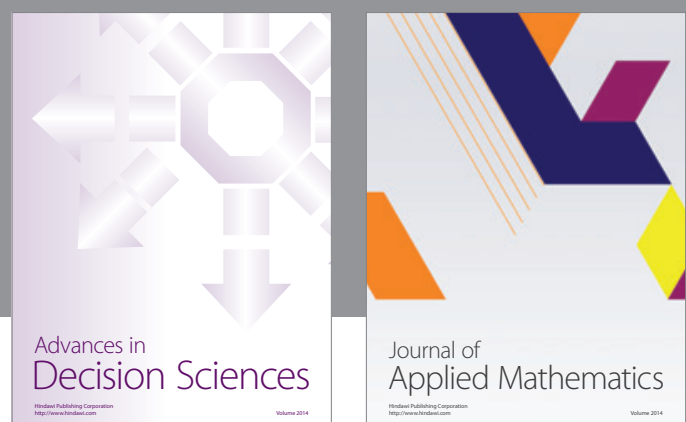

Journal of

Applied Mathematics
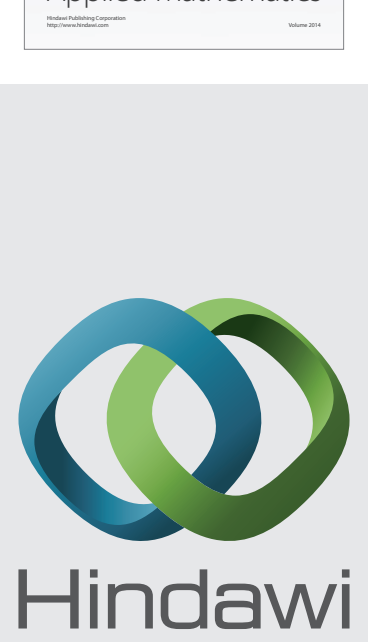

Submit your manuscripts at http://www.hindawi.com
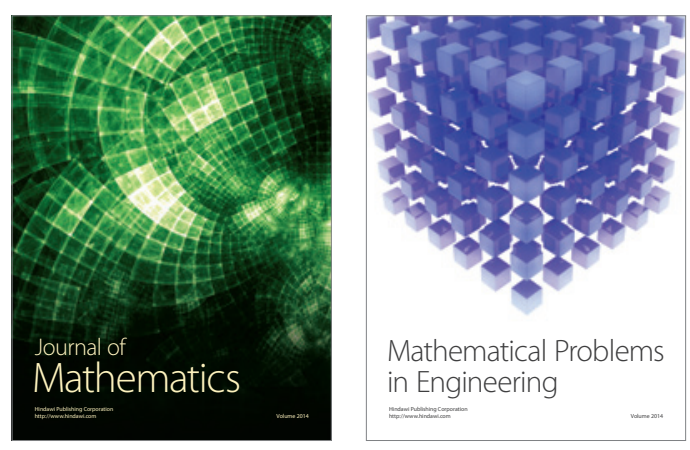

Mathematical Problems in Engineering
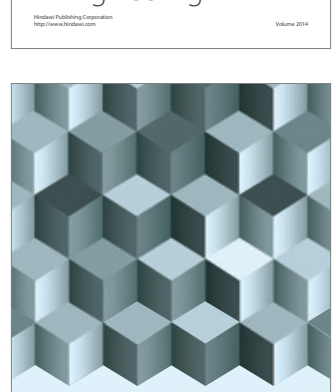

Journal of

Function Spaces
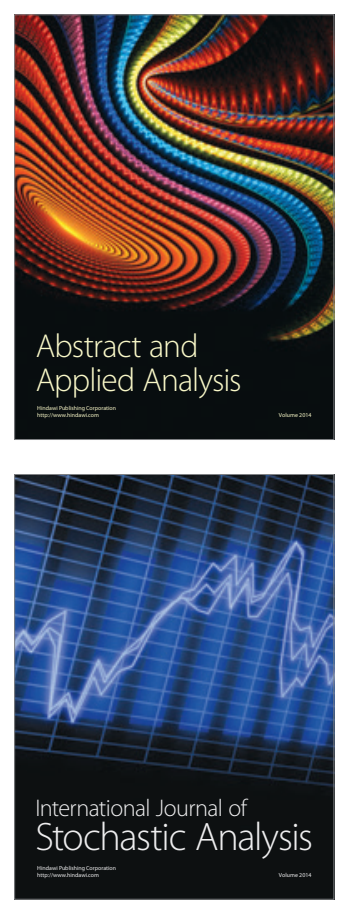

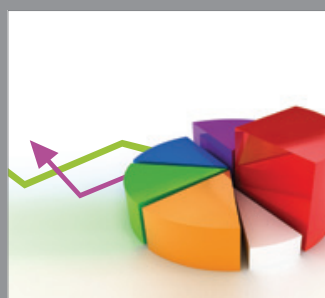

ournal of

Probability and Statistics

Promensencen
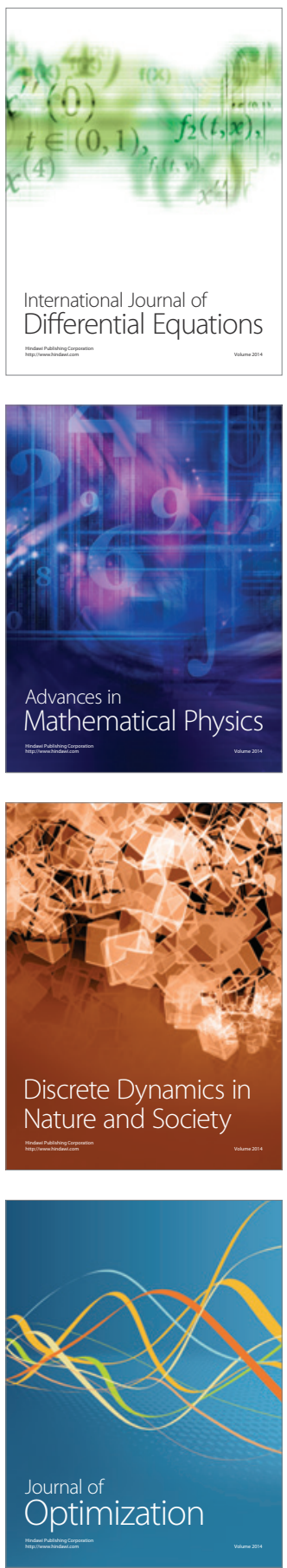